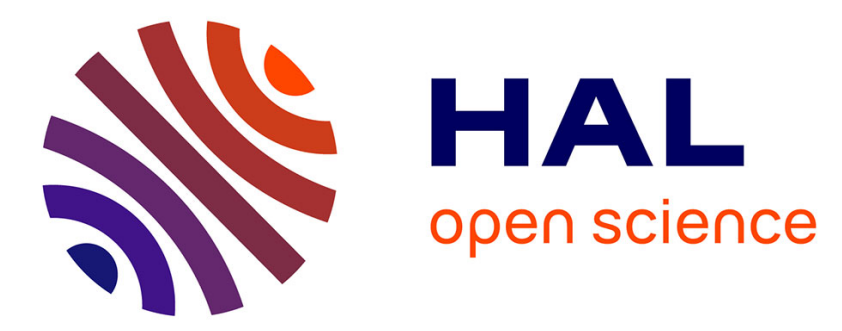

\title{
An improved Lagrangian thermography procedure for the quantification of the temperature fields within polycrystals
}

Rian Seghir, Jean-Francois Witz, Laurence Bodelot, Eric Charkaluk, Philippe Dufrenoy

\section{To cite this version:}

Rian Seghir, Jean-Francois Witz, Laurence Bodelot, Eric Charkaluk, Philippe Dufrenoy. An improved Lagrangian thermography procedure for the quantification of the temperature fields within polycrystals. Quantitative InfraRed Thermography Journal, 2013, 10 (1), pp.74-95. 10.1080/17686733.2013.785207 . hal-00871131

\section{HAL Id: hal-00871131 \\ https://hal.science/hal-00871131}

Submitted on 27 Jul 2021

HAL is a multi-disciplinary open access archive for the deposit and dissemination of scientific research documents, whether they are published or not. The documents may come from teaching and research institutions in France or abroad, or from public or private research centers.
L'archive ouverte pluridisciplinaire HAL, est destinée au dépôt et à la diffusion de documents scientifiques de niveau recherche, publiés ou non, émanant des établissements d'enseignement et de recherche français ou étrangers, des laboratoires publics ou privés.

\section{(c) (1) $\$$}

Distributed under a Creative Commons Attribution - NonCommerciall 4.0 International 


\title{
An improved lagrangian thermography procedure for the quantification of the temperature fields within polycrystals
}

\author{
Rian Seghir ${ }^{\text {a,b,c }}$, Jean-François Witz ${ }^{\mathrm{a}, \mathrm{b}, \mathrm{c}}$, Laurence Bodelot ${ }^{\mathrm{d}}$, Eric Charkaluk ${ }^{\mathrm{a}, \mathrm{b}, \mathrm{c} *}$ and \\ Philippe Dufrénoy ${ }^{\mathrm{a}, \mathrm{c}, \mathrm{e}}$ \\ ${ }^{a}$ Univ Lille Nord de France, Lille, France, ${ }^{b}$ ECLille, LML, Villeneuve d'Ascq, France; ${ }^{c} C N R S$, UMR \\ 8107, Villeneuve d'Ascq, France; ${ }^{d}$ Ecole Polytechnique ParisTech, LMS, UMR 7649, Palaiseau, \\ France; ${ }^{e}$ USTL, LML, Villeneuve d'Ascq, France
}

\begin{abstract}
Polycrystalline metallic materials are made of an aggregate of grains more or less well oriented with respect to the loading axis. During mechanical loading, the diversity of grain orientations leads to a heterogeneous deformation at the local scale. It is well known that most of the plastic work generated during the deformation process reappears in the form of heat, whereas a certain proportion remains latent in the material and is associated with microstructural changes. To access the local stored energy during deformation processes, experimental energy balances are needed at a suitable scale. Thus, simultaneous measurements of thermal and kinematic fields were made in-house at the microstructural scale of a 316L stainless steel submitted to a macroscopic monotonic tensile test. The aim of the present study is to propose a complete calibration strategy allowing us to estimate the thermal variations of each material point along its local and complex deformation path. This calibration strategy is a key element for achieving experimental granular energy balances and has to overcome two major experimental problems: the dynamics of each infrared focal plane array sensor that leads to undesired spatial and temporal noise and the complexity of the local loading path that must be captured by simultaneous complementary measurement. The improvement of such a multifield strategy is crucial for performing properly the experimental and local energy balances required to build new energetically based damage criteria.
\end{abstract}

Keywords: polycrystal; thermo-mechanical coupling; infrared camera; calibration

\section{Introduction}

Since the beginning of the last century, many studies have been conducted regarding the plastic deformation processes in metallic materials in order to improve the global understanding of the deformation mechanisms, of the elastic-plastic transition, of the deformation localisation and, obviously, of the damage processes leading to ultimate failure. One can cite, for example, the pioneering works of Schmid [1], Sachs [2], von Mises [3] and Taylor [4].

In this context, thermal effects associated with the deformation process because of thermomechanical couplings have been brought to light and the evolution and transformation of the mechanical energy have been studied. It is now well known that during cold working, most of the plastic work developed during the deformation reappears as heat, whereas a certain

*Corresponding author. Email: eric.charkaluk@ec-lille.fr 
proportion remains latent in the material. The latter, classically called stored energy, is associated with microstructural changes and could, therefore, constitute a judicious indicator of damage evolution. As a consequence, experimental energy balances are necessary. An extensive review of the early work in this field was published by Bever et al. [5] including the pioneering works of Taylor et al. [6-8].

However, most energy balances presented in the literature were performed at a macroscopic scale, thus only dealing with the macroscopic appearance of inelastic processes. One can cite works on necking, Lüders bands, the Portevin-Le Chatelier phenomenon, dynamic deformation and shear bands [9-16]. Consequently, there is a lack of information on energetics regarding the local deformation processes in heterogeneous material like polycrystals. Such information is necessary in order to study microstructurally based phenomena such as microplasticity or fatigue. The literature reports only a few works in this research area: a study concerning the monotonic tensile deformation of an A1050 Al bi-crystal [17], one on an oligocrystal (seven grains) of a commercial pure aluminium [18] and finally one concerning an AISI 316L stainless steel polycrystal (2000 grains) [19]. As microstructutural thermal effects and energy balances are the keystone of these studies, simultaneous measurements of local temperature and displacement fields are required. In fact, to express the infrared (IR) scene in its undeformed configuration, it is necessary to follow the radiative flux associated with each material point of the observed zone during the deformation process before moving it back to the original configuration. This constitutes the concept of 'Lagrangian thermography'. This task is relatively complex and so is the subsequent energy balance computation. The main difficulties lie in the need for accurate local thermal information and in the necessity to follow each material point during the experiment so as to evaluate its temperature.

To our knowledge, there is no work dealing with the entire procedure necessary to obtain, from the digital levels (DL) provided by the IR camera, a temperature field within each grain of a metallic polycrystal along its deformation path. In particular, the three main problems at this scale are: (1) no uniform emissivity, (2) the calibration method and (3) the temperature data processing. The objective of this work is to propose such a comprehensive procedure. This paper comprises three main parts. In the first part, data used in this study are presented. In the second, a calibration procedure is developed to reach a fine estimation of the temperature of all material points along their deformation path at the specimen's surface. Finally, we present an application of the calibration method to the analysis of the thermal fields obtained on an A316L polycrystals subjected to a monotonic tensile test.

\section{Simultaneous measurements of microstructural kinematic and thermal fields}

\subsection{Review of techniques}

To our knowledge, only four experimental techniques of combined in situ calorimetric and kinematic measurements at the microstructural scale of metals have been developed during the last few years. These techniques will be detailed in this section, but it is important to note that each of them introduces specific constraints and limitations regarding the studied material and the fineness of the microstructure.

The constraint of QIRT $^{1}$ and $\mathrm{DIC}^{2}$ (or any other method for displacement measurement) seems to be a priori opposite. On the one hand, QIRT requires knowledge of the specimen's emissivity. The problem is classically solved by using a coating of uniform and known IR emission such as a black mat paint. On the other hand, DIC requires a very heterogeneous coating, in terms of gray levels, for material point tracking. Several ways of achieving such a coupling are presented here: 
(1) Two-face measurement: Each imaging system (CCD and IR camera) observes one face of the specimen. This technique was used, for example, in [18]. Its main advantage is that QIRT and DIC constraints are separated. In the case of [18], a thin, opaque and uniform black paint is sprayed onto one face to obtain a thermal emissivity close to 1 , while a microgrid is applied on the other face to measure the displacement field. Note that the microgrid could be replaced by a speckle coating. If thermal and kinematic fields are sought for comparison at the microstructural scale, this technique requires the microstructure to be the same on both faces, which is only the case if the grains are columnar and extruded through the thickness. Therefore, this technique is only suitable for thin specimens of single, bi-, or oligocrystals ( $<10$ grains) but not for standard polycrystalline microstructures where both faces are different.

(2) One-shot measurement: A single IR camera is used to obtain both kinematic and thermal fields on the same surface and over the same space and time discretisation. In this case, the contrast of IR images due to emissivity heterogeneity is exploited to perform DIC analyses and thereby obtain reliable displacement fields. This technique, called 'one-shot measurement', involves modification of the classic DIC algorithm in order to add the temperature evolution in the optical flow resolution. It was recently introduced in $[20,21]$. The first main advantage of this technique is that any bias in time and space matching between the kinematic and thermal fields are avoided. The second is its relatively simple experimental implementation compared to other techniques. However, this technique yields a strong limitation on the spatial resolution since the pixel size of the best IR camera is currently about 10 times larger than the one of the best CCD camera. As a consequence, local gradients or discontinuities in the kinematic and thermal fields are smoothed before being analysed. Because of the resolution of currently available IR cameras, this technique seems to be much more adapted to aggregates whose grain size is greater, at best, than $200 \mu \mathrm{m}$.

(3) Single-face measurement: Both imaging systems (CCD and IR camera) observe the same zone at the same instant. Two kinds of set-up could be considered:

- the introduction of an angle between the IR and CCD cameras. This technique was used in [17] and requires a correction of the distortions introduced by the set-up in the experimental fields. Hence, using a high-magnification lens like the $G_{1}$ (a high magnification lens which provides a geometrical spatial resolution equal to the IR Focal Plane Array (IRFPA) sensor size) for IR microscopy in such a configuration would be impossible since its small depth of field $(\approx 100 \mu \mathrm{m})$ would limit to $1^{\circ}$ the maximum allowable angle between the IR camera and the normal relative to the sample's surface. Therefore, this technique is much more suitable for large grain samples, such as the bi-crystal investigated in [17], than for small grain samples requiring optical and IR microscopy.

- the use of a dichroic mirror (see Figure 1(a) and 1(b.b)). This technique has been used in [19]. Thanks to its filtering properties, the dichroic mirror transmits the IR radiations (wavelength between 2 and $6 \mu \mathrm{m}$ ) toward the IR camera located in front of the sample and reflects the rest of the radiations, including the visible radiations, towards the CCD camera which is perpendicular to the sample's surface. The main disadvantages of this set-up are its difficult implementation and the experimental bias due to the mirror (transmission, reflections). Nevertheless, in this case, both arrays of detectors remain parallel to the image of the sample's surface, thus preventing any distortion in the measured fields. 
(a)

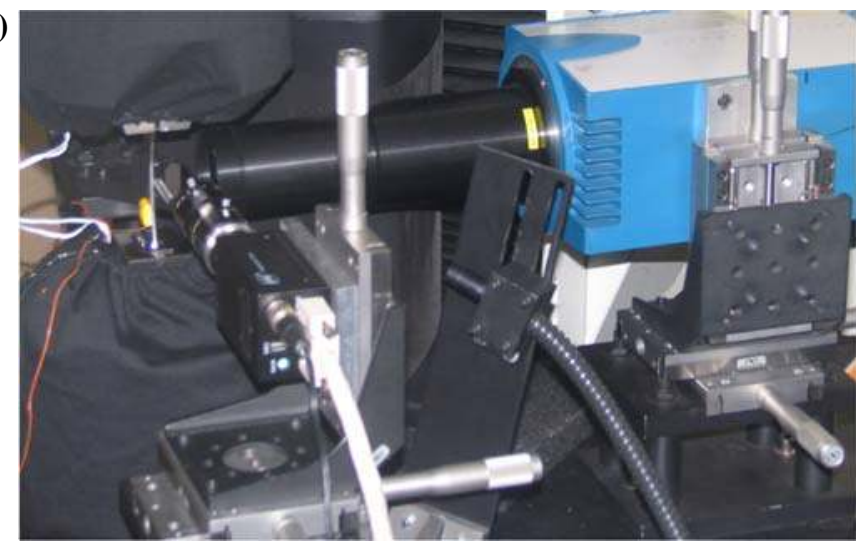

(b)
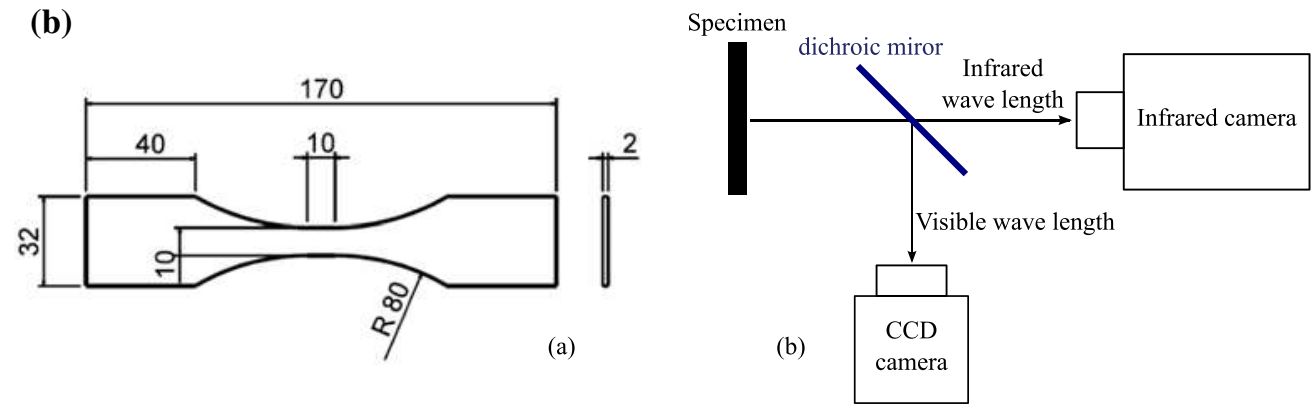

Figure 1. (a) View of the experimental set-up (from [19]); (b.a) Shape and dimensions of the specimen; (b.b) Schematic representation of the set-up.

In both cases, a specific coating has to be developed. It must be as homogeneous as possible in the IR spectrum and heterogeneous, in terms of gray levels, in the visible one [19]. For better accuracy, the heterogeneous emission properties of the coating have to be identified.

\subsection{Focus on our 'single-face' experimental set-up}

The experimental set-up and data on which our study is based were introduced in [19]. The main steps of the fully coupled thermomechanical measurements are recalled and some assumptions, in particular on the calibration procedure, are detailed. Displacement fields are obtained, thanks to DIC measurements performed with Correli ${ }^{\mathrm{Q}}$ developed at the 'Laboratoire de Mécanique et Technologie' (LMT) in Cachan (France) [22]. Images of the loaded sample are grabbed by a Jai CV-M4 + CCD camera whose detector matrix is $1368 \times 1024$. It is used with a Tamron 23FM50SP $50 \mathrm{~mm}$ lens and extension tubes, leading to a spatial resolution of $6.5 \mu \mathrm{m} \times 6.5 \mu \mathrm{m}$ per pixel and a field of view of $8.9 \times 6.7 \mathrm{~mm}^{2}$. Since the DIC computation is performed with $16 \times 16$ pixels subsets, the spatial resolution of the displacement and strain fields data is finally $104 \mu \mathrm{m} \times 104 \mu \mathrm{m}$. Temperature fields are obtained by IRT. The sample is filmed by a focal plane array (FPA) Cedip Jade III MWIR camera whose detector matrix is $320 \times 240$. It is used with a high magnification lens called $G_{1}$. The spatial resolution for the temperature fields data is, therefore, $30 \mu \mathrm{m} \times 30 \mu \mathrm{m}$. 
Different steps are necessary to obtain fully coupled measurements of kinematic and thermal fields at the grain scale of a polycrystal. Since details of each step can be found in [19], only the main features are recalled here:

(1) Dichroic mirror: as previously described, a dichroic mirror is located in front of the sample, at a $45^{\circ}$ angle with the specimen and facilitates simultaneous use of the IR and CCD cameras to observe the same zone.

(2) Pixel-to-pixel calibration: provided that each detector of an FPA camera has its own dynamics, each detector is calibrated regardless of its neighbours in a defined range of temperature.

(3) Coating: the key feature of the fully coupled measurements lies in the coating applied on the sample. On the one hand, the coating exhibits a speckle aspect at a very fine scale, covering a wide range over the gray scale, which is required for the DIC computation. On the other hand, this coating exhibits, at the working scale, a high and uniform emissivity close to the one of the black body (BB) used for calibration, which is necessary for performing IRT.

(4) Lagrangian configuration: the thermal fields are measured on a deforming body. The DIC displacement data are, therefore, used to track the displacement of the material points in order to express the temperature fields in the undeformed configuration.

Finally, data presented in [19] are fully coupled insofar as both fields correspond to the same zone at the same time and are expressed in the undeformed configuration. Moreover, the spatial resolutions achieved in the strain measurements as well as in the temperature measurements are suitable for providing meaningful data at the scale of the grains of the material studied.

We propose here some improvements to the method presented in [19]. In the following, we detail the entire procedure necessary to convert the DL provided by the IR camera facing the deforming specimen into temperature fields of the specimen gauge area expressed in the Lagrangian configuration.

\section{Eulerian thermal metrology}

Following the thermal evolution of a material point during a mechanical loading is not a trivial task:

(1) the IR camera does not measure temperature but radiative flux and does not express it in physical units but in DL,

(2) the measurement could be affected by optical distorsions,

(3) each sensor of the IRFPA camera has its own dynamics and noise,

(4) the relationship between radiative flux and temperature depends on the surface and volume properties of the specimen such as emissivity, diffusivity ...

(5) external heat sources could dramatically affect the observed thermal scene.

Therefore, it is essential to establish a calibration strategy that minimises the error at each step. This part focuses on the calibration steps that must be conducted in the Eulerian configuration. 


\subsection{Thermal calibration}

\subsubsection{Pixel to pixel}

The IR camera expresses the received flux (emitted by an object) in DL. To convert these DLs into physical units, it is necessary to calibrate each pixel/sensor of the IRFPA camera. Here, a 'flux calibration' method is proposed. Its principle is to determine, thanks to a least squares method, the optimal function $\phi$ to convert the DL into flux emitted by the observed object at fixed integration time IT of the camera. Contrary to a temperature calibration, the function $\phi$ does not depend on the emissivity of the observed object. Finally, as each IRFPA sensor of the camera has its own dynamics, this calibration must be done for each detector (pixel-to-pixel calibration [23]). This is the main advantage of this proposal.

Knowing different uniform temperatures $T_{k}$ at the surface of an extended BB and their corresponding DL $\left(\mathrm{DL}_{k}^{i}\right)$ at each pixel $(i)$ of the IR camera sensor matrix, one can build a calibration curve for each pixel/sensor. The procedure is performed according to the following equation:

$$
\Phi\left(\mathrm{IT}, T_{k}, i\right)=\sum_{u=1}^{p} \alpha_{u}\left(\mathrm{DL}_{k}^{i}\right)^{u}
$$

where coefficients $\alpha_{u}$ are determined by the least squares method and $p$ corresponds to the degree of the classic polynomial calibration law. Equation (1) is actually a system of $k$ equations to be minimised for every calibration temperature $T_{k}$ of the $\mathrm{BB}$, related to the flux by the following equation:

$$
\Phi=\varepsilon \sigma T^{4}
$$

where $\phi, \epsilon$ and $\sigma$ are, in the present case, the flux radiated by the $\mathrm{BB}$ at fixed temperature $T$, the uniform emissivity of the $\mathrm{BB}\left(\varepsilon_{\mathrm{BB}}=0.97\right)$ and the Boltzmann constant $\sigma=5.67032 \times 10^{-8} \mathrm{Wm}^{-2} \mathrm{~K}^{-4}$ respectively.

In practice, in order to limit the temporal noise of each sensor, the calibration is done, for each $T_{k}$, with a temperature field corresponding to the mean field over $100 \mathrm{IR}$ pictures. In the present case, the minimisation was performed, thanks to Matlab.

Figure 2(a) presents the flux calibration functions for all the sensors of the IRFPA camera. They consist of 6th-order polynomial functions and $k$ is equal to 14 , the number of temperatures recorded during our calibration. One could note that they constitute a set of curves with a maximum deviation of $3000 \mathrm{DL}$ at a given homogeneous emitted flux and that some pixels have a flat response as a function of the flux. These pixels are considered defective and replaced by the mean value of their direct neighbours. Once the thermal field is recovered using a pixel-to-pixel calibration (Digital Level to flux) and then the Botlzman law (Equation (2), flux to temperature), a comparison between the measured thermal field and the uniform one of the observed $\mathrm{BB}$ could be done in order to estimate the accuracy of the calibration method.

Figure 2(b) presents the mean and extreme deviations between the effective temperature of the $\mathrm{BB}$ and the 'measured' or calibrated one. It shows that the mean error for each pixel, when the flux radiated by the object remains in the [405-465] $\mathrm{W} \mathrm{mm}^{-2}$ domain, fluctuates from -10 to $10 \mathrm{mK}$ and that the absolute error is locally inferior to $30 \mathrm{mK}$. Note that such a result remains valid for the presented flux domain regardless of any temporal noise.

It is relatively important to underline that the camera is now calibrated for a specific range of flux and not for a range of temperature. Note also that, contrary to the $\mathrm{BB}$, in the case of 

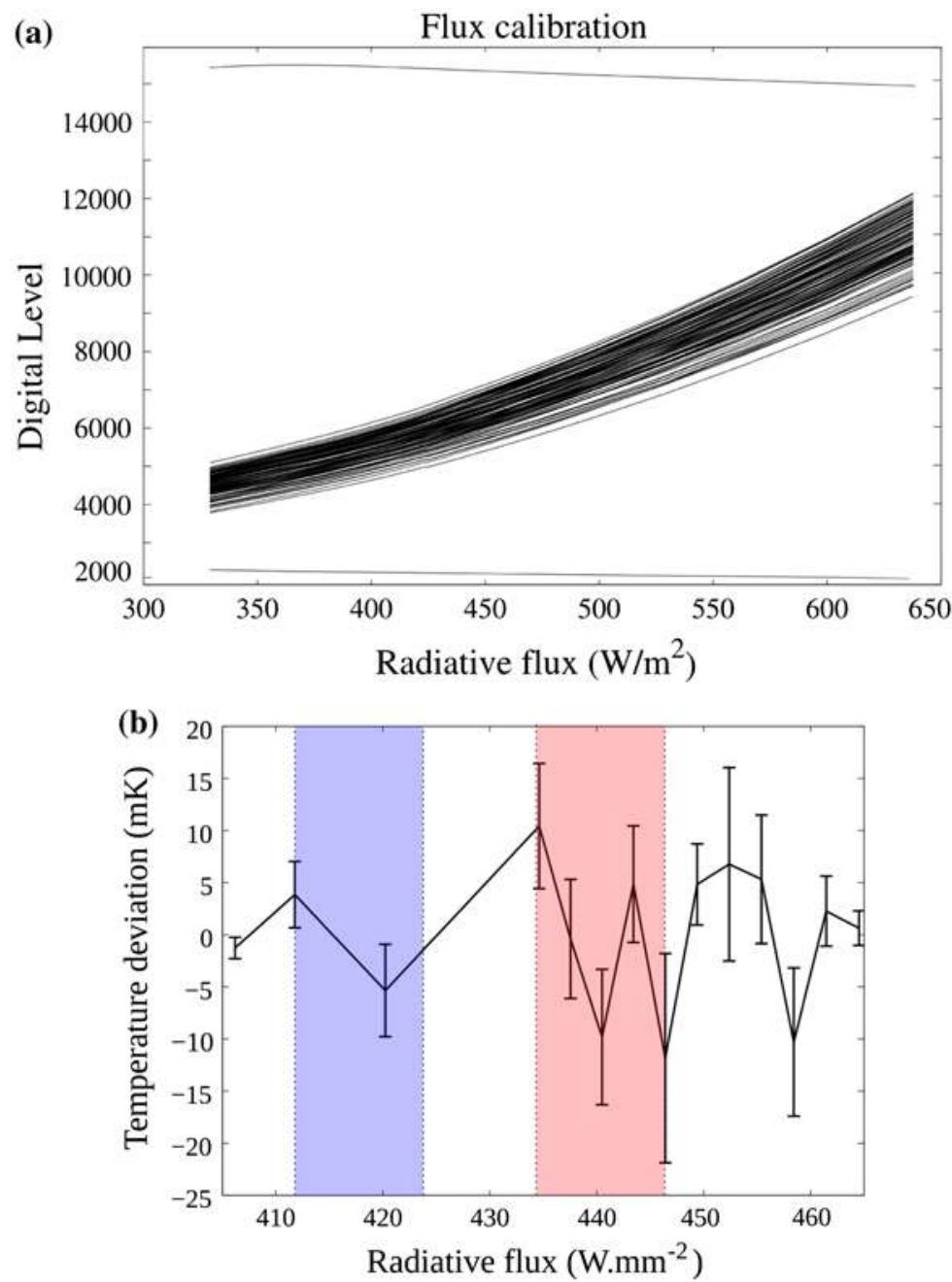

Figure 2. (a) 76,800 flux calibration functions associated to each IRFPA sensor and (b) error between radiative flux and measured temperature $(\mathrm{mK})$ for the $k=14$ temperatures.

the tested specimen introduced in Section 4.3, we know that the coating emissivity is actually not a constant. According to [24], the coating standard deviation is about $9 \times 10^{-3}$ with a mean value of 0.927 . In Figure 2(b), two domains are highlighted, thanks to coloured bands: the left band is the flux domain corresponding to the interval $\left[25-27^{\circ} \mathrm{C}\right]$ when the mean specimen emissivity $\left(\varepsilon_{s}=0.927\right)$ is taken into account; the right band corresponds to the same interval $\left[25-27^{\circ} \mathrm{C}\right]$, but this time the $\mathrm{BB}$ emissivity $\left(\varepsilon_{\mathrm{BB}}=0.97\right)$ is taken into account. Obviously, the interval $\left[25-27^{\circ} \mathrm{C}\right]$ does not lead to the same range of flux, whether the BB or the specimen is considered, because of their difference in emissivity. It is, therefore, important to get the best possible estimation of the specimen emissivity in order to determine the suitable range of calibration flux, especially in the case of a specimen emissivity far from one. An other possibility, if the coating emissivity were unknown prior to calibration, would be to extend the calibration range while making sure it is large enough to account for the difference in emissivity between the $\mathrm{BB}$ and the sample. The crucial point here would be to 
remain well below the calibration limit so as to avoid reaching the domain where the calibration error dramatically increases because of the polynomial fitting.

According to Figure 2(b) and considering the interval $\left[25-27^{\circ} \mathrm{C}\right]$ corresponding to the specimen's temperature variation during the monotonic tensile test ${ }^{3}$ considered in this study, one could conclude that the present calibration remains valid for a coating emissivity that would range from 0.92 to 0.98 . Thus, the calibration range seems sufficient to take into account the effective emissivity distribution [24].

\subsubsection{Temporal noise}

As previously mentioned, the calibration functions are obtained from averages over 100 IR fields at each given BB temperature. Nevertheless, during an experiment, these functions are applied to single IR pictures of the specimen taken at a measurement frequency of $140 \mathrm{~Hz}$. Consequently, the effective measurement uncertainty will necessarily increase. To check if the global uncertainty remains below $30 \mathrm{mK}$, the pixel-to-pixel calibration is applied to obtain 100 IR thermal fields of 14 different fixed temperature scenes recorded at $140 \mathrm{~Hz}$. Then, for each of the 14 temperature scenes, the maximal local deviation from the effective temperature over the 100 fields is recorded. Figure 3 presents these local maximal deviations from the effective temperature for the 14 different BB temperatures as different data processings were applied: (1) the solid curve corresponds to the thermal deviation considering full frequency $(140 \mathrm{~Hz})$ and full resolution, (2) the dashed curve shows the thermal deviation considering lower frequency $(25 \mathrm{~Hz})$ and full resolution and (3) the dashed and dotted curve presents the thermal deviation considering low frequency $(25 \mathrm{~Hz})$ and low resolution, i.e. the average over $3 \times 3$ pixels as classically done [25]. Note that this low-frequency signal is obtained by using a low-pass filter in the Fourier space. One can note that the curve denoted in (3) is the only one leading to a measurement uncertainty under $30 \mathrm{mK}$. In fact, it has been shown that the spatial resolution of an IR imaging system is not equal to the size of the area observed by the IRFPA pixel. The method used to determine the real measurement resolution, called slit response function, is described in [25] and shows that for the CEDIP Jade III camera, a zone

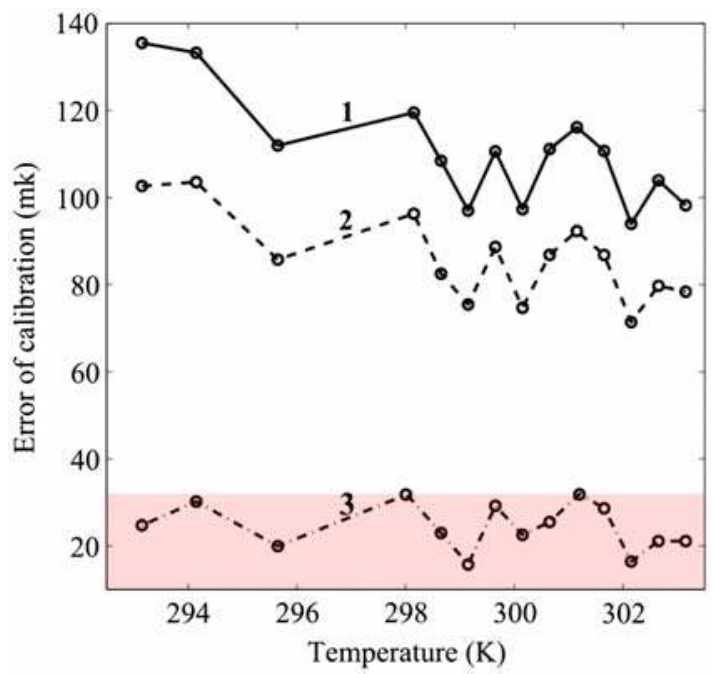

Figure 3. Error on calibration (mK) for different cases: (1) $140 \mathrm{~Hz}$ and full resolution, (2) $25 \mathrm{~Hz}$ and full resolution, and (3) $25 \mathrm{~Hz}$ and average over $3 \times 3$ pixels. 
of interest (ZOI) of $3 \times 3$ pixels is sufficient to capture $100 \%$ of emitted radiations. Taking into account this additional constraint, this implies that the minimal resolution provided by the IR imaging system is $90 \times 90 \mu \mathrm{m}(3 \times 3$ pixels $)$ which has been used in [24]. In this work, a projection-based approach has been preferred on spatial domains greater than $3 \times 3$ pixels. This will be detailed in Section 4.2.

In conclusion, Figure 3 shows that when both constraints are verified (low-pass filter at $25 \mathrm{~Hz}$ and average over $3 \times 3$ pixels), the highest local deviation from the effective temperature of the $\mathrm{BB}$ is at worst $30 \mathrm{mK}$. Note that the low-pass filter will be applied on the Lagrangian signal and not directly on the Eulerian one. This point will be detailed in Section 3.3.3. Finally, the thermal signal must be considered as having a frequency of $25 \mathrm{~Hz}$, a resolution of $90 \mu \mathrm{m} \times 90 \mu \mathrm{m}(3 \times 3$ pixels $)$ and a maximum uncertainty of $30 \mathrm{mK}$.

\subsubsection{Flux decomposition}

In order to perform proper quantitative IR thermography at a local scale, two phenomena have to be taken into account. The first one is related to the reflections that could appear even if the test is performed in a totally controlled environment. In the present case, the fully coupled measurements are performed with a dichroic mirror. It is, unfortunately, a preferential source of reflections. Considering that the IR camera is fixed, this first phenomenon is Eulerian (reflection spots do not move when the specimen deforms). The second phenomenon is the spatial variation of emissivity and diffusivity due to the thermal and mechanical properties of the coating. Considering again that the IR camera is fixed, this second phenomenon is Lagrangian (the 'apparent emissivity' field moves and deforms with the specimen). The two above-mentioned perturbations have to be dealt with before determining and analysing the thermal fields. However, evaluating experimentally the reflections and the emissivity field is not a simple task. The objective of this part is to show that both aspects can be treated numerically since they are not connected. In the present case, reflections and 'apparent emissivity' affect the observed field at totally different spatial scales. A reasonable physical assumption is to consider that the reflections affect the field at low spatial frequency (LSF) and are Eulerian, whereas the 'apparent emissivity' field affects the observed field at high spatial frequency (HSF) and is Lagrangian (see Figure 4(b)). To treat both aspects, one focuses on a time period $A$ as shown in Figure 4(a). During this period, the specimen is around the thermal equilibrium: the specimen is not loaded yet and its temperature is known and uniform. At this time, the local and/or global spatial flux variations are only linked to the reflections (environment) and the apparent emissivity (coating). Figure 4(a) zone $B$ presents the mean thermal response of the specimen during the tensile test.

Let us decompose the flux captured by the IRFPA sensors. The flux measured by the IR camera $\phi$ is the difference between the observed flux $\phi_{\mathrm{obs}}$ and the flux radiated by the IRFPA camera sensor $\phi_{\text {cam }}$ cooled at $T_{\text {cam }}=77 \mathrm{~K}$.

$$
\left\{\begin{array}{l}
\phi=\phi_{\mathrm{obs}}-\phi_{\mathrm{cam}} \\
\phi_{\mathrm{obs}}=\phi_{\mathrm{obj}}+\phi_{\mathrm{refl}} \\
\phi_{\mathrm{cam}}=\sigma T_{\mathrm{cam}}^{4}
\end{array}\right.
$$

The observed flux $\phi_{\mathrm{obs}}$ is composed of two terms: the flux really emitted by the observed object $\phi_{\text {obj }}$ and some possible reflection fluxes $\phi_{\text {refl }}$ caused by external heat sources and by the fact that the emissivity is not equal to one. In the case of a gray body, the flux emitted by the object follows the Boltzman law as introduced in Equation (4): 


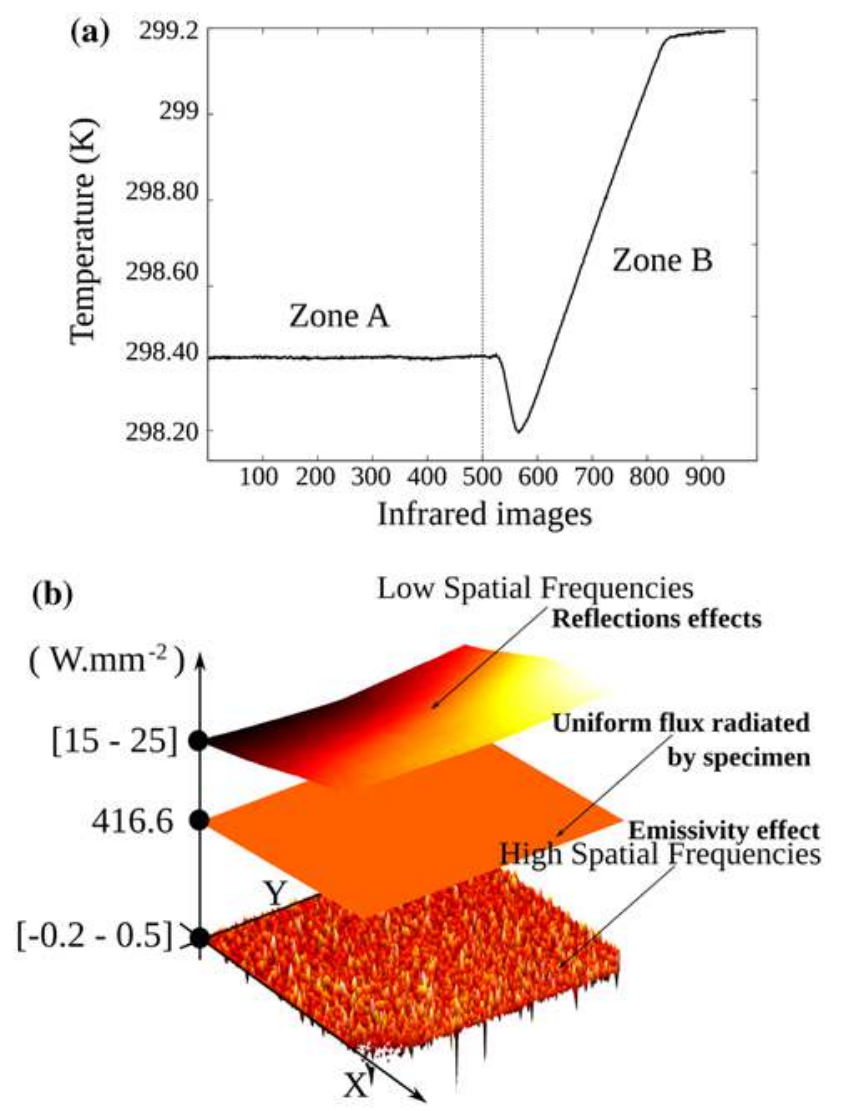

Figure 4. (a) Mean temperature evolution over the specimen gauge section during a monotonic tensile test and (b) flux decomposition in low spatial frequencies, high spatial ones and uniform ones.

$$
\phi_{\mathrm{obj}}=\epsilon_{\mathrm{obj}} \sigma T_{\mathrm{obj}}^{4}
$$

where $\epsilon_{\mathrm{obj}}$ and $T_{\mathrm{obj}}$ are, respectively, the emissivity field and the temperature field at the specimen's surface. Combining Equations (3) and (4), one could write:

$$
\phi=\underbrace{\epsilon_{\mathrm{obj}} \sigma T_{\mathrm{obj}}^{4}}_{(\mathrm{HSF}, \mathrm{LSF})}+\underbrace{\phi_{\text {refl }}-\sigma T_{\mathrm{cam}}^{4}}_{\mathrm{LSF}}
$$

The main interest of this decomposition is to highlight the separation of variables previously mentioned. Analysing the LSF of $\phi$ by spectral decomposition, one could write:

$$
\left.\phi\right|_{\mathrm{LSF}}=\bar{\epsilon}_{\mathrm{obj}} \sigma \bar{T}_{\mathrm{obj}}^{4}+\phi_{\mathrm{refl}}-\sigma T_{\mathrm{cam}}^{4}
$$

with $\left.\phi\right|_{\text {LSF }}$ is the measured flux without high spatial frequencies. HSF phenomena are assumed here if the spatial period is lower than $500 \mu \mathrm{m}$ i.e. lower than $10 \%$ of the picture. Within this assumption, the LSF signal does not take into account any local variations. $\bar{\epsilon}_{\text {obj }}$ is the measured mean emissivity of specimen's surface (obtained by indirect emissivity measure- 
ment [24]), $\bar{T}_{\text {obj }}$, the mean temperature (measured by thermocouple) of the specimen's surface during the time period $A$ (see Figure 4). Figure 4(b) presents the radiative flux decomposition introduced in Equation (5) and induced by the 'apparent emissivity'. The HSF signal fluctuates from -0.2 to $0.5 \mathrm{~W} \mathrm{~mm}^{-2}$; it results from the high-frequency part of $\epsilon_{\mathrm{obj}} \sigma T_{\mathrm{obj}}^{4}$ in Equation (5). The LSF signal fluctuates from 15 to $25 \mathrm{~W} \mathrm{~mm}^{-2}$; it corresponds to $\phi_{\text {refl }}$ in Equation (5) and the constant signal from the term $\bar{\epsilon}_{\mathrm{obj}} \sigma \bar{T}_{\mathrm{obj}}^{4}-\sigma T_{\mathrm{cam}}^{4}$ in Equation (6). Its value is $416.6 \mathrm{~W} \mathrm{~mm}^{-2}$.

Equation (6) leads to:

$$
\phi_{\text {refl }}=\left.\phi\right|_{\mathrm{LSF}}-\bar{\epsilon}_{\mathrm{obj}} \sigma \bar{T}_{\mathrm{obj}}^{4}+\sigma T_{\mathrm{cam}}^{4}
$$

Combining Equations (7) and (5), it is now possible to determine the apparent emissivity field of the specimen at a thermal steady-state.

$$
\epsilon_{\mathrm{obj}}=\frac{\phi-\left(\left.\phi\right|_{\mathrm{LSF}}-\bar{\epsilon}_{\mathrm{obj}} \sigma \bar{T}_{\mathrm{obj}}^{4}\right)}{\sigma \bar{T}_{\mathrm{obj}}^{4}}
$$

Classically, in coupled-field measurements where heterogeneous coating (for DIC) is necessary, emissivity is considered constant and homogeneous [26,27]. Firstly, it is not such a rude assumption in most cases. Secondly, no direct and in situ method for emissivity field measurement exists, even if some authors tried to obtain it by observing the specimen in two distinct spectral bands [28]. Finally, it is worth mentioning that most QIRT analyses do not attempt to assess such a precise and local surface temperature. Using Equation (8), one can build a posteriori the temperature error field induced by the hypothesis of homogeneous emissivity. This error is corrected in the present case. Figure 5(a) presents the temperature error field and the corresponding emissivity built from Equation (8). The temperature error field is built from the difference between the field obtained with a uniform emissivity and the one obtained with the apparent emissivity map (see Equation (8)). One can note that here, the classic assumption of homogeneous emissivity leads to an absolute range of more than $200 \mathrm{mK}$ of thermal spatial noise, which is clearly connected to the heterogeneity of the coating shown in the visible wavelength in Figure 5(b). The mean value of the temperature error field is null but its standard deviation is $23 \mathrm{mK}$. The built emissivity map yields to limit this temperature error. Regarding the emissivity field (see Figure 5(a) 2nd colour bar), it ranges from 0.92 to 0.93 with a mean value of 0.927 (corresponding to the experimental measure) and a standard deviation of $1 \times 10^{-3}$ (the experimental measure was $9 \times 10^{-3}$ ). In comparison with the experimental data provided by [24], one can conclude that the standard deviation of the coating emissivity is underestimated here even though the mean value is conserved. Nevertheless, the error on the final thermal field is locally reduced here and remains widely lower than the measurement uncertainty.

It is important to mention that emissivity and diffusivity depend partially on the temperature of the specimen. However, the apparent emissivity field previously built does not take any variation of temperature into account since it is built around the equilibrium state. Nevertheless, the variation of temperature is, in the present case, i.e. a monotonic tensile test up to a mean axial strain of $2.5 \%$, lower than $1 \mathrm{~K}$. Thus, one neglects the effect of temperature on the emissivity map during the test. Before applying the emissivity field established here to the flux in order to recover the temperature through the Boltzman law (see Equation (4)), the IR scene will need to be expressed first in its undeformed configuration. 
(a)
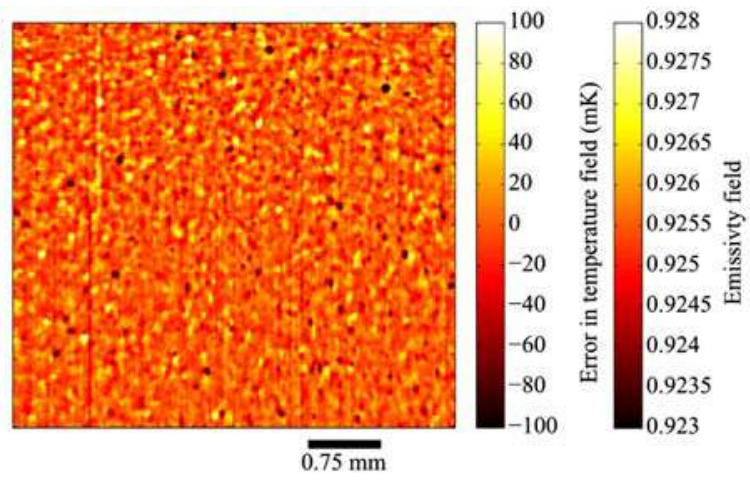

(b) 2.5

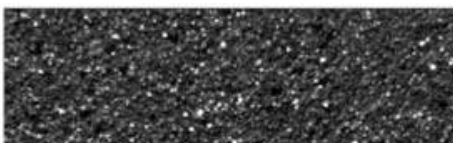

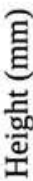

$-2.5$

$-2.5$

0

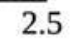

Width (mm)

Figure 5. (a) Temperature error field with homogeneous emissivity assumption (0.927) considering non homogeneous emissivity field as reference and corresponding apparent emissivity field. (b) Coating in visible wavelength and zoom on a specific millimetric grain.

\subsection{Digital image correlation}

In this study, kinematic fields are obtained from visible images of the sample taken by the $\mathrm{CCD}$ camera and subsequently processed by the digital image correlation software Correli ${ }^{Q 4}$ [29]. The change in DIC software compared to Bodelot's works [24] was justified, on the one hand, by the appearance of some artifacts in the displacement field, clearly associated with the correlation method and, on the other hand, by the fact that the finite element formalism of Correli ${ }^{Q 4}$ facilitates a numerical/experimental dialogue since the displacement variations follow similar local shape functions. The basis of the algorithm is the comparison between two images of the same specimen area at two different states of the loading: a reference one and a deformed one. It is expressed as follows:

$$
\begin{aligned}
& x_{i}-x_{0}=u_{0}(A) \\
& y_{i}-y_{0}=v_{0}(A)
\end{aligned}
$$

where $A\left(x_{i}, y_{i}\right)$ are the coordinates of point $A$ in the deformed configuration, $A\left(x_{0}, y_{0}\right)$ are the coordinates of point $A$ in the reference configuration and $\underline{U}_{0}^{i}\left(u_{0}^{i}(A), v_{0}^{i}(A)\right)$ are the axial and 
transversal displacements of a material point $A$ expressed in the reference configuration. Obviously, the specimen must exhibit a sufficient speckle texture in terms of gray levels. This speckle aspect can naturally exist on a material observed at high magnification [30], but can also be artificial as in the present case.

As the principle of DIC can be found in [31], we only recall here the main results in terms of resolution, uncertainty, and shape functions.

- Correli ${ }^{Q 4}$ assumes a bilinear kinematics within 4-noded square elements. This is the assumption classically made in finite element formulation and the corresponding elements are called $Q 4 \mathrm{P} 1$, i.e. 4-node elements using first degree polynomials as interpolation functions.

- Following [24], a $16 \times 16$ pixel subset window was chosen as ZOI. Thus, the kinematic resolution is $104 \mu \mathrm{m} \times 104 \mu \mathrm{m}$, since the imaging system initially provides a resolution of $6.5 \mu \mathrm{m} \times 6.5 \mu \mathrm{m}$ per pixel.

- The uncertainty on the displacements calculation for a given experiment and a given DIC software mainly depends on two points: (1) the coating, i.e. its random aspect and the gray level domain it covers and (2) the ZOI size: the larger the ZOI size, the greater the accuracy but the smaller the spatial resolution. The procedure to estimate the uncertainty in relation with the ZOI size and the coating is already integrated in Correli $^{Q 4}$ and has been used for our test with a $16 \times 16$ pixel ZOI. The uncertainty on the displacements is then given by Correli ${ }^{Q 4}$ after a standard estimation procedure. It is close to $6 \times 10^{-3}$ pixels or $4 \times 10^{-2} \mu \mathrm{m}$.

\subsection{Lagrangian metrology and thermography}

To express the IR scene in its undeformed configuration, it is necessary to follow the radiative flux associated with each material point of the observed zone before moving it back in the reference configuration. This task is carried out, thanks to the displacement fields obtained by DIC. It constitutes the concept of 'Lagrangian thermography'. For this, a time and space matching between the kinematic and thermal fields has to be done.

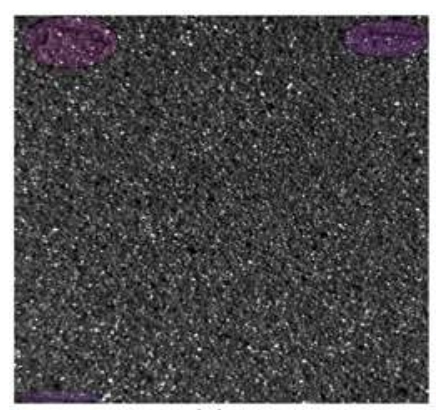

(a)

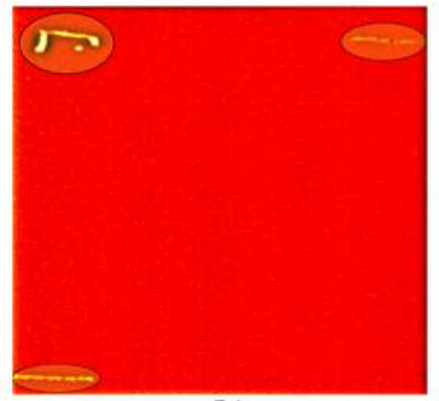

(b)

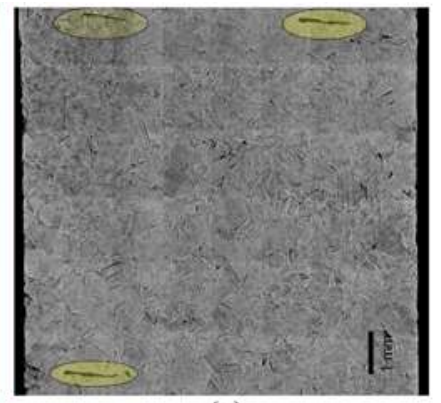

(c)

Figure 6. Visualisation of the marks on (a) an optical image (CCD camera), (b) on an IR image (IR camera) and (c) on a micrograph performed after the test and removing the coating. 


\subsubsection{Time and space matching}

Kinematic and IR measurements are first synchronised in space by using some marks made on the specimen that are observable in both visible and IR wavelengths. These marks are presented in Figure 6. They allow us to rotate, translate and stretch the fields in order to precisely match each of them at the initial instant. A time synchronisation is obtained by triggering the beginning of the acquisition made by both cameras, which will thereafter run at their own frequencies. In order to use a unique spatio temporal mesh for all data, it was decided to take advantage of the high resolution of the complementary microstructural field coming from the electron backscatter diffraction (EBSD), described in Section 4.3 and of the high frequency of the IR camera. Thus, one assumes a spatio temporal interpolation of the kinematic and IR field on:

- the EBSD mesh for space,

- the IR grid for time.

It constitutes a trilinear interpolation (2D in space, 1D in time) of data, which is implemented in Matlab using the interp3 function. Note that this procedure does not change the resolution of any of the quantities since they are only interpolated linearly on a unique reference mesh.
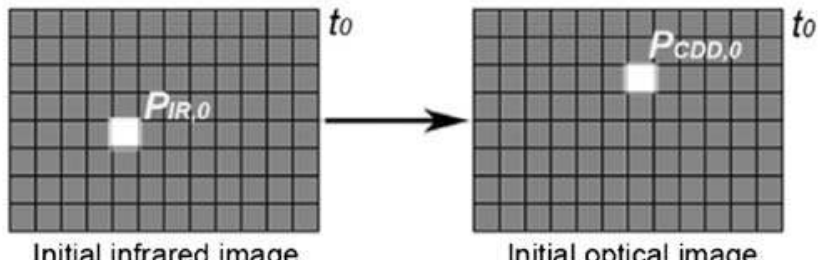

Initial optical image
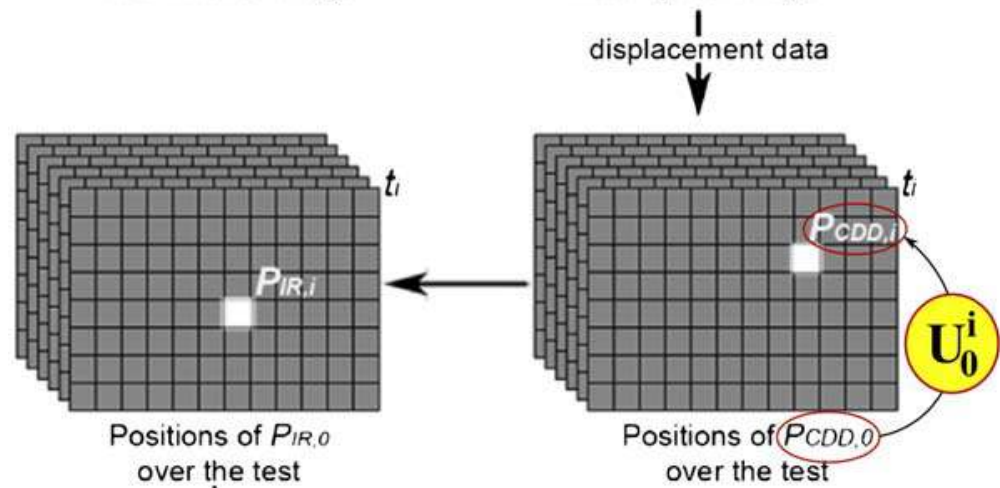

Positions of PIR,O

over the test

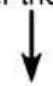

over the test

Infrared movie in the reference configuration

Figure 7. Principle of the algorithm used to follow material points moving in front of the IR sensor and to IR fields in the reference configuration (Lagrangian thermography) from [26]. 


\subsubsection{Lagrangian thermography}

Figure 7 recalls the principle of the Lagrangian thermography as presented in [26]. $P_{\mathrm{IR}, i}$ denotes a point in the IR camera coordinate system expressed in the current configuration, $i$. e. at time $t_{i}$ and $P_{\mathrm{CCD}, i}$ a point in the CCD camera coordinate system in the same configuration. Note that $P_{\mathrm{CCD}, 0}$ and $P_{\mathrm{IR}, 0}$ are in the same coordinate system, thanks to space and time synchronisations and to spatio-temporal linear interpolation (see previous section). Then, the displacement between instant 0 and $t_{i}$, expressed in the initial configuration and denoted $\underline{U}_{0}^{i}$, can be directly applied to $P_{\mathrm{IR}, i}$. Following Equations (9) and (10), one obtains:

$$
P_{\mathrm{IR}, 0}=P_{\mathrm{IR}, i}-\underline{U}_{0}^{i}
$$

Equation (11) has to be applied to each material point $P_{\mathrm{IR}, i}$ at each time $t_{i}$. Finally, the displacement, radiative flux and emissivity fields are expressed in the same reference system.

\subsubsection{Temporal noise correction}

The temporal noise that appears in IR fields (see Figure 4) is mainly due to the variation of flux in the room during the test and to the intrinsic noise of each pixel. Thus, it has to be removed before applying the emissivity correction and determining the temperature fields. The first possibility is to remove the intrinsic noise of each detector. However, it is not an easy task as a detector is traversed by different material points during the deformation of the specimen. Reversely, within the Lagrangian thermography framework, as a material point passes through different detectors during the deformation of the specimen, the material point associated with noise will change with the time. However, even if each material point is affected by the sensitivity of each pixel along its deformation path, the signal evolution is the one of a thermoelastoplastic deformation process. Thus, in order to perform a time smoothing with a more physical sense, the treatment has to be done after the Lagrangian thermography on each material point. Different kinds of data processing strategies could be considered here:

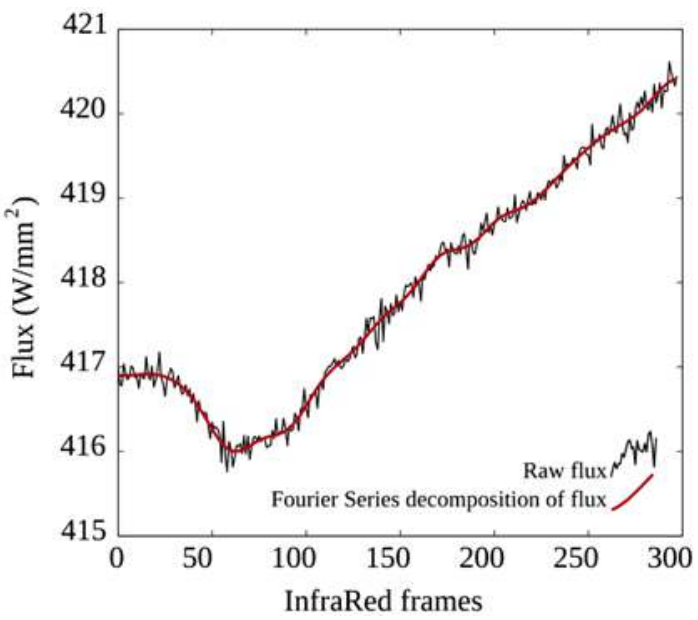

Figure 8. Raw and time smoothed $(>25 \mathrm{~Hz})$ radiative flux of one material point within the centre zone of the specimen gauge area. 
- A polynomial function (of 6th degree at least). It would represent quite well the drop and subsequent increase of temperature but not the initial and final evolutions (see Figure 4). Moreover, every rapid variation of radiative flux would be removed and the consequence would be a global uniformity of the thermal response.

- A function carrying a physical sense. A constitutive law would have to be postulated and its parameters identified with the help of the strain fields provided by DIC.

- A classic low-pass filter that could be applied in order to remove high time frequencies in Fourier space.

As introduced in Section 3.1, a low-pass filter cutting frequencies strictly higher than $25 \mathrm{~Hz}$ has already been chosen. Such a low-pass filter guarantees that the thermal uncertainty remains low. Figure 8 presents the raw and smoothed radiative flux evolutions of one of the material points within the centre zone of the specimen. One can observe that the main variations of radiative flux are conserved in this case.

\subsubsection{Apparent emissivity correction}

In Section 3.1.3, a local spatial variation of flux response was identified on the unloaded specimen. The nature of this flux distribution was quantified and attributed to the emissivity distribution of the coating. Then, in Section 3.3.2, Lagrangian thermography was performed on IR scenes in order to express both fields in the same initial configuration. Now, the apparent emissivity correction can be applied on the whole IR scene to finally express the effective surface temperature of the specimen from $\phi_{\mathrm{obj}}$.

$$
T_{\mathrm{obj}}=\left(\frac{\phi_{\mathrm{obj}}}{\sigma \epsilon_{\mathrm{obj}}}\right)^{\frac{1}{4}}
$$

with $\epsilon_{\mathrm{obj}}$ introduced in Section 3.1.3. The calibrated thermal scene expressed in the reference configuration will be analysed in the next section using the additional microstructural data.

\section{Microstructural thermal fields on an AISI 316L austenitic stainless steel specimen}

\subsection{Material and test}

The main features of the test analysed in this section are described in details in [19]. The material studied is an AISI 316L austenitic stainless steel. A dog-bone-shaped flat sample 2$\mathrm{mm}$ thick (see Figure 1(b.a)) was heat-treated under air for $2 \mathrm{~h}$ at $1200^{\circ} \mathrm{C}$ and waterquenched immediately after. A displacement-controlled monotonic tensile test was applied to the sample with a constant strain rate of $5.10^{-3} \mathrm{~s}^{-1}$, which induces a spatial mean strain of $2.5 \%$ and a nominal stress equal to $250 \mathrm{MPa}$ close to the end of the test [26]. Fully coupled kinematic and thermal fields measurements were performed during the monotonic test at the microstructure scale. The studied fields correspond to a $5 \mathrm{~mm} \times 5 \mathrm{~mm}$ area in the centre of the specimen. They will be denoted as calibrated fields once they have undergone all the numerical treatments proposed in the previous sections. In addition to these kinematic and thermal measurements, an EBSD analysis was performed with a Jeol 6100 scanning electron microscope using a conventional electron gun operating at $25 \mathrm{kV}$ with a probe current of the order of $1 \mathrm{nA}$. Data analysis was performed with the OIM software provided by TSL with a pitch of $20 \mu \mathrm{m}$. 


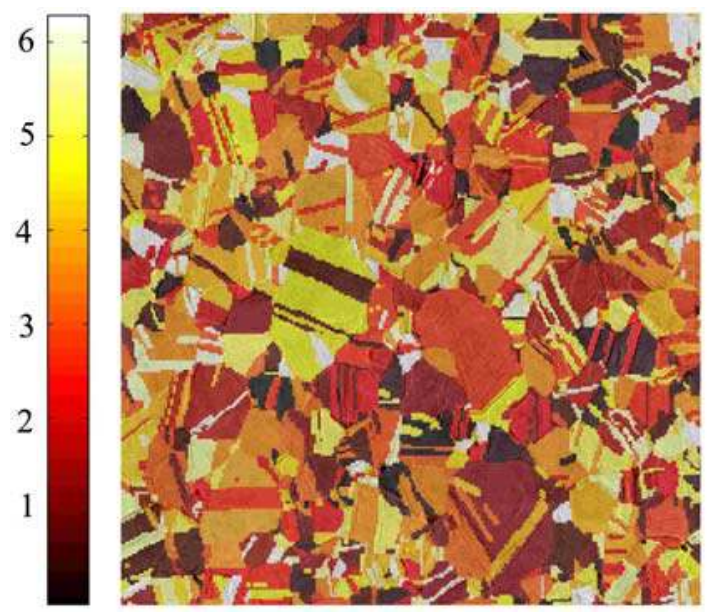

Figure 9. First angle $\phi_{1}$ of the triplet $\left(\phi_{1}, \Phi, \phi_{2}\right)$ provided by the EBSD analysis (in rad), in the global frame of the analysed area. The EBSD is superimposed onto the micrograph of the specimen obtained after the test and presented in Figure 6(c).

Figure 9 presents the EBSD field of the 1st angle $\phi_{1}$ of the Euler angle triplet $\left(\phi_{1}, \Phi, \phi_{2}\right)$, which characterises the $3 \mathrm{D}$ orientation of the material points in the global frame of the analysed area after the test. This field presents the mean grain orientation per grain. The EBSD analysis shows that, in the $5 \mathrm{~mm} \times 5 \mathrm{~mm}$ central area, the microstructure is composed of 1776 grains with a $118 \mu \mathrm{m}$ mean grain size (measurement based on diameter approximation). Thus, the mean grain size of the tested sample matches the spatial resolution capabilities of both measurement techniques. However, $60.7 \%$ of the grains have a diameter lower than $100 \mu \mathrm{m}$, but this only represents $15.1 \%$ of the studied area. Hence, more than $84 \%$ of the ZOI is suitable for full-field measurements analysis in order to observe heterogeneities. In particular, approximately 20 grains possess a grain size greater than $400 \mu \mathrm{m}$, which makes an intragranular investigation possible.

\subsection{Field projection on microstructure}

In order to analyse all the available fields (temperature, displacements, crystallographic orientations ...) and to obtain deformation and thermal flux fields by derivation of the primal ones, we proposed to use an original method presented in detail in [32]. The principle is to project the primal fields (temperature and displacements) on the specimen microstructure (grains) known through the EBSD analysis. These grains constitute a natural set of continuous and smooth spatio-temporal domains separated by grain boundaries where kinematic and thermal continuities are not necessarily ensured. For displacements, this is confirmed by complementary profilometry analyses done on the specimen after the test [32], and for temperature, this is justified by some studies on the Kapitza effect $[33,34]$ that are related to the thermal resistance associated with grain boundaries in polycrystals.

This method is based on the assumption that, at the first order, the temperature and displacement fields measured at the surface of the sample are more related to the grains emerging at the surface than to the grains found in depth. This is mainly due to the 
deformation mechanisms that are much more confined and limited in depth than on a free surface.

A minimisation, in the sense of the least squares method, between the calibrated thermal field and a polynomial function is done within each grain, regardless of its neighbours, at each time increment. A second-order polynomial projection basis is then chosen for both displacements and for the temperature within each grain. The main consequences on the resulting fields are the following:

(1) Biparabolic displacement field: in the present case, the main plastic deformation mechanism is the slip activation on particular slip systems. Displacement fields within each grain and at each time increment are projected on a biparabolic base and as strain is calculated from the displacement gradient, we implicitly assume here a bilinear strain field within grains. As many grains of the $5 \mathrm{~mm} \times 5 \mathrm{~mm}$ central area exhibit a single slip system activation, this assumption is relatively consistent even if it certainly leads to larger errors where multiple slip systems activation is observed. Moreover, this assumption also leads to a significant reduction of variables: six parameters per grain at each instant, which represents 12,000 variables per field instead of 62,500. The intragranular strain map is, therefore, obtained by a direct analytical derivation [32].
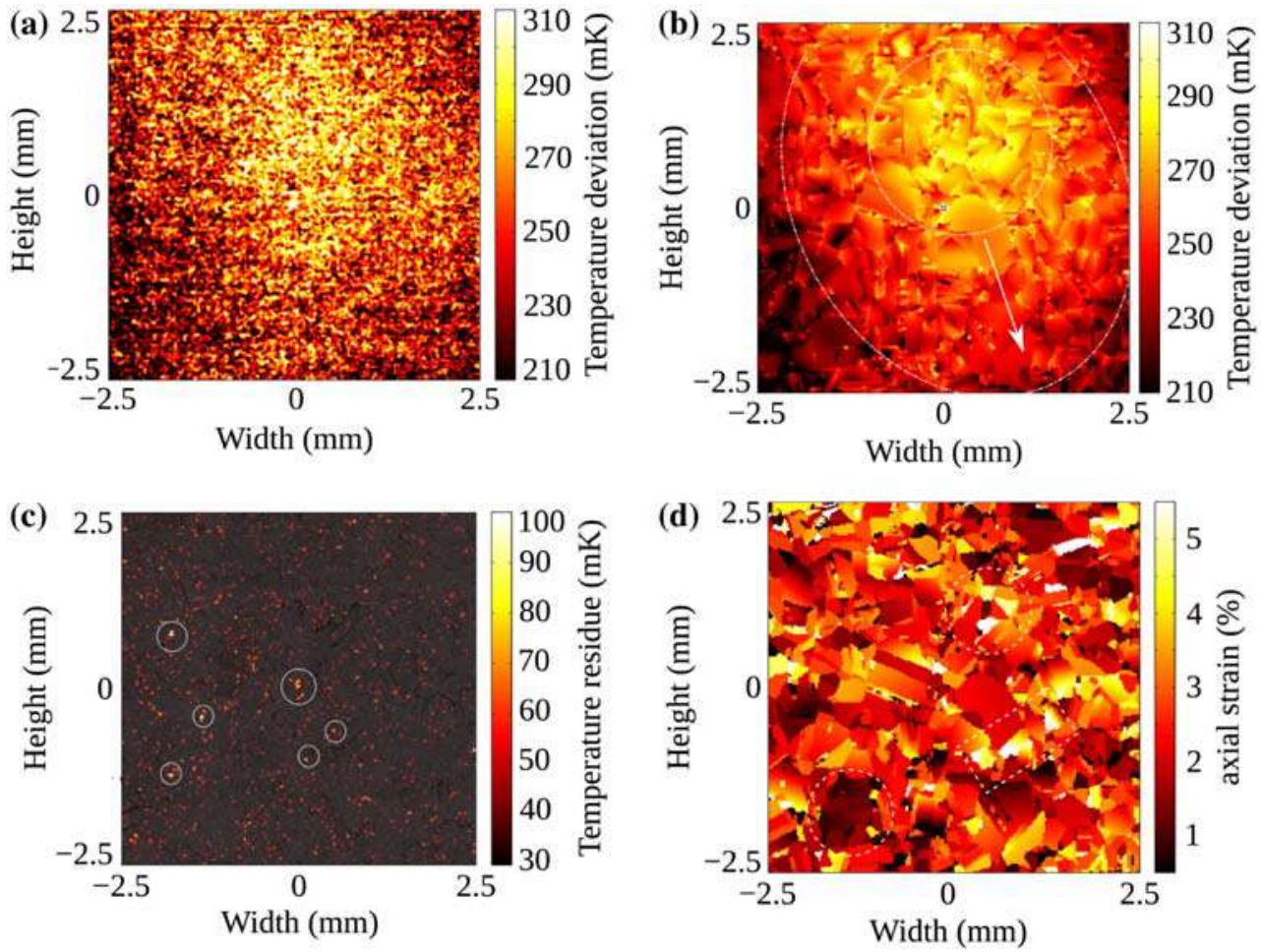

Figure 10. (a) the original temperature field $(\mathrm{mK}),(\mathrm{b})$ the projected one $(\mathrm{mK})$, (c) the residual value of the temperature field after projection $(\mathrm{mK})$ and $(\mathrm{d})$ the axial strain map $(\%)$. They all correspond to a $250 \mathrm{MPa}$ nominal stress. 
(2) Biparabolic thermal field: as conductive fluxes are calculated from the temperature Laplacian, uniform conductive exchanges within grains at each instant are implicitly assumed through this hypothesis. Since every local heat source initiated within the grains quickly becomes uniform owing to conductive phenomena, it is assumed that conductive exchanges are instantaneously uniform within the grain and on its boundaries. Therefore, as for the displacement fields, the thermal field can be analytically derived.

In the case of grains with a size lower than the spatial resolutions, fields are projected on lower order polynomial functions. However, one could point out that more than $84 \%$ of the studied area contains grains whose size is greater than the spatial resolution. Moreover, it has to be pointed out that no data are lost in this projection procedure. The whole decomposition is additive. Residual fields between calibrated fields and projected ones are complementary and can be analysed separately. In the present case, it was verified that the standard deviation on temperature residual values is almost constant and corresponds to $24 \mathrm{mK}$, which is lower than the measurement uncertainty. As previously mentionned, the classical averaging operation over $3 \times 3$ pixels (see Section 3.1.2) is not necessary here since the above-mentioned projection conserves the average and covers, in the present case, larger areas (grains).

\subsection{Some results}

Figure 10(a) and (b) presents the calibrated temperature field and the projected one at $250 \mathrm{MPa}$ nominal stress.

One can clearly observe a smoothed field in Figure 10(b) and the contribution of each grain to the global field can now be identified and measured. It exhibits a $100 \mathrm{mK}$ thermal gradient from a specific area toward the gauge section borders, which clearly reflects a conductive phenomenon from the centre-circled area towards the specimen border. Note that the hot region is not centred within the gauge section, which is certainly the sign of a thermal localisation due to the microstructure and not to the specimen geometry. This is confirmed on the strain map presented in Figure 10(d), which corresponds to a nominal stress of $250 \mathrm{MPa}$ and a spatial mean strain of $2.5 \%$. This map shows a correlation between the higher strained area (close to 7\%) and the hotest zone. However, remember that the strain is a cumulative variable on time, whereas the temperature is an instantaneous one. A simple point to point correlation is, therefore, difficult. The available calibrated data consist of the displacement and temperature fields (as illustrated in Figure 10) over time. As the data are analytically projected (see Section 4.2), differentiating them in order to determine strains and thermal flux fields is now possible. One can also underline certain thermal discontinuities on grain boundaries, notably around the big grain at the centre. Even if the projection method naturally accentuates the presence of such discontinuities, raw thermal data show that many of these discontinuities do exist at grain or twin boundaries and that their values are over the experimental error on temperature measurements [35]. Indeed, if one considers the residual values of the temperature field (see Figure 10(c)), where a threshold corresponding to the temperature error $(30 \mathrm{mK})$ has been applied, one can observe that the maximum values are generally inside grains. Analysis has shown that these residual values remain quite low and stationary in space during the test. Then, these discontinuities could be related to the Kapitza effect [33]. Moreover, if one considers the central big grains in Figure 10(b), one can see intragranular thermal heterogeneities. Although they could be related to the crystal plastic behaviour associated with different slip systems, further analyses are under progress at the grain scale. 


\section{Conclusion}

In this paper, a complete metrological procedure was introduced in order to obtain in situ fully coupled kinematic and thermal fields expressed in the reference configuration. Special attention was given to the decomposition of the measurement in errors:

- a Eulerian part related to the sensors dynamics,

- a Eulerian part related to reflections due to the environment and the experimental set-up,

- a Lagrangian part related to the intrinsic emission properties of the specimen's surface.

The metrological procedure was applied to the digital level and gray level frames recorded by IR and CCD cameras, respectively, in [19]. The spatial resolution of the final thermal fields is $104 \mu \mathrm{m} \times 104 \mu \mathrm{m}$, which matches the mean grain size of the specimen $(\approx 120 \mu \mathrm{m})$. The uncertainty of measurement is lower than $30 \mathrm{mK}$ and the frequency of the phenomena that can be captured reaches $25 \mathrm{~Hz}$. Finally, such calibrated fields were used to observe the thermal evolution of a stainless steel flat specimen gauge section at the scale of its grains. This analysis was performed assuming a projection of the thermal fields on the specimen's microstructure. Observations reveal a very high heterogeneity of the thermal fields at this scale as well as an early thermal localisation clearly connected to the high deformations observed at the surface of the specimen. Although the goal of this study was the determination of microstructural granular thermal fields, this technique paves the way to the potential achievement of energy balances at the grain scale of polycrystals.

\section{Acknowledgements}

This study has been funded by the CNRS, the Nord-Pas-de-Calais area (CISIT) and the European Community (FEDER). The authors would like to acknowledge the CNRS Research Group GDR's 2519 'Full-field measurements and Identification in Solid Mechanics' for fruitful discussions.

\section{Notes}

1. Quantitative InfraRed Thermography.

2. Digital Image Correlation.

3. This interval has been verified a posteriori by thermocouple measurement.

\section{References}

[1] Schmid E. Yield point of a crystals: critical shear stress law. In: Proceedings of the 1st International Congress Applied Mechanics; 1924; Delft, Netherlands, p. 342.

[2] Sachs G. Zur ableilung einer fleissbedingung [About the derivation of a yield criterion]. Zeitschrift des Vereins Deutscher Ingenieure. 1928;72:734-6.

[3] von Mises R. Mechanik der plastischen formänderung von kristallen [Mechanics of crystals plastic deformation]. Zeitschrift für angewandte Mathematik und Mechanik. 1928;8(3):161-85.

[4] Taylor GI. Plastic strains in metals. Journal of the Institute of Metals. 1938;62:307-24.

[5] Bever MB, Holt DL, Titchener AL. The stored energy of cold work. Progress in Material Sciences. 1973;17:1-192.

[6] Farren WS, Taylor GI. The heat developed during plastic extension of metals. Proceedings of the Royal Society A. 1925;107(743):422-51.

[7] Taylor GI, Quinney H. The latent heat remaining in a metal after cold working. Proceedings of the Royal Society of London. 1934;143(849):307-26.

[8] Taylor GI, Quinney H. The latent heat remaining in a metal after cold working. Proceedings of the Royal Society of London. 1937;163(913):157-81.

[9] Wattrisse B, Muracciole J, Chrysochoos A. Thermomechanical effects accompanying the localized necking of semi-crystalline polymers. International Journal of Thermal Sciences. 2002;41(5):422-7. 
[10] Chrysochoos A, Wattrisse B, Muracciole J, Kaim YE. Field of stored energy associated with localized necking of steel. Journal of Mechanics of Materials and Structures. 2009;4(6):245-62.

[11] Louche H, Chrysochoos A. Thermal and dissipative effects accompanying lüders band propagation. Materials Science and Engineering A. 2001;307(1-2):15-22.

[12] Ait-Amokhtar H, Fressengeas C, Boudrahem S. The dynamics of Portevin-Le Châtelier bands in an $3.2 \% \mathrm{Mg}$ alloy from infrared thermography. Materials Science and Engineering A. 2008;488(12):540-6.

[13] Ranc N, Wagner D. Experimental study by pyrometry of Portevin-Le Châtelier plastic instabilities type a to type b transition. Materials Science and Engineering A. 2008;474(1-2):188-96.

[14] Guduru P, Rosakis A, Ravichandran G. Dynamic shear bands: an investigation using high speed optical and infrared diagnostics. Mechanics of Materials. 2001;33(7):371-402.

[15] Padilla H, Lambros J, Beaudoin A, Robertson I. Spatiotemporal thermal inhomogeneities during compression of highly textured zirconium. Experimental Mechanics. 2011;51:1061-73. 10.1007/ s11340-010-9425-4. Available from: http://dx.doi.org/10.1007/s11340-010-9425-4

[16] Zehnder A, Guduru P, Rosakis A, Ravichandran G. Million frames per second infrared imaging system. Review of Scientific Instrumentation. 2000;71(10):3762-8.

[17] Saai A, Louche H, Tabourot L, Chang H. Experimental and numerical study of the thermomechanical behavior of Al bi-crystal in tension using full field measurements and micromechanical modeling. Mechanics of Materials. 2010;42(3):275-92.

[18] Badulescu C, Grédiac M, Haddadi H, Mathias J, Balandraud X, Tran H. Applying the grid method and infrared thermography to investigate plastic deformation in aluminium multicrystal. Mechanics of Materials. 2011;43(1):36-53.

[19] Bodelot L, Sabatier L, Charkaluk E, Dufrénoy P. Experimental setup for fully coupled kinematic and thermal measurements at the microstructure scale of an AISI 316L steel. Materials Science and Engineering A. 2009;501(1-2):52-60.

[20] Silva ML, Ravichandran G. Combined thermoelastic stress analysis and digital image correlation with a single infrared camera. Journal of Strain Analysis for Engineering Design. 2011;46 (1):783-93.

[21] Maynadier A, Poncelet M, Lavernhe-Taillard K, Roux S. One-shot measurement of thermal and kinematic fields: infrared image correlation (IRIC). Experimental Mechanics. 2012;52(3):241-55.

[22] Hild F, Raka B, Baudequin M, Roux S, Cantelaube F. Multiscale displacement field measurements of compressed mineral-wool samples by digital image correlation. Applied Optics. 2002;41 (32):6815-28.

[23] Berthel B. Mesure thermographiques de champs de dissipation accompagnant la fatigue à grand nombre de cycles des aciers [Thermographic measurements of dissipative fields accompanying the HCF of steels] [PhD thesis]; 2007. Available from: http://tel.archivesouvertes.fr/tel-00410074

[24] Bodelot L. Etude couplées des champs cinématiques et thermiques à l'échelle de la microstructure des matériaux métalliques [Coupled study of kinematic and thermal fields at the microstructure scale of metallic materials] [PhD thesis]; 2008. Available from: http://tel.archives-ouvertes.fr/tel00347987

[25] Pron H, Bissieux C. Focal plane array infrared cameras as research tools. QIRT Journal. 2004;1 (2):229-40.

[26] Bodelot L, Charkaluk E, Sabatier L, Dufrénoy P. Experimental study of heterogeneities in strain and temperature fields at the microstructural level of polycrystalline metals through fully-coupled full-field measurements by digital image correlation and infrared thermography. Mechanics of Materials. 2011;43(11):654-70.

[27] Dumoulin S, Louche H, Hopperstad O, Børvik T. Heat sources, energy storage and dissipation in high-strength steels: experiments and modelling. European Journal of Mechanics - A/Solids. 2010;29(3):461-74.

[28] Kasem H, Thevenet J, Boidin X, Siroux M, Dufrenoy P, Desmet B, Desplanques Y. An emissivitycorrected method for the accurate radiometric measurement of transient surface temperatures during braking. Tribology International. 2010;43(10):1823-30.

[29] Hild F, Roux S. Correlli Q4: a software for finite-element displacement field measurements by digital image correlation, internal report 269. (Tech Rep). LMT Cachan. ENS Cachan, France; 2008.

[30] El Bartali A, Aubin V, Degallaix S. Surface observation and measurement techniques to study the fatigue damage micromechanisms in a duplex stainless steel. International Journal of Fatigue. 2009;31(11-12):2049-55. 
[31] Sutton M, Orteu JJ, Schreier H. Image correlation for shape, motion and deformation measurements. New York: Springer; 2009.

[32] Seghir R, Witz JF, Charkaluk E, Dufrénoy P. Improvement of thermomechanical full-field analysis of metallic polycrystals by crystallographic informations. Mechanics and Industry. 2012; Forthcoming.

[33] Kapitza P. Heat transfer and superfluidity of helium II. Physical Review. 1941;60(4):354-5.

[34] Nan C, Birringer R. Determining the Kapitza resistance and the thermal conductivity of polycrystals: a simple model. Physical Review B. 1998;57(14):8264-8.

[35] Seghir R. Experimental and numerical investigation of thermomechanical couplings and energy mbalance in metallic polycrystals [PhD thesis]. Ecole Centrale de Lille [in English]; 2012. 